\title{
Assessment of Uplink Time Difference Of Arrival (U-TDOA) Position Location Method in Urban Area and Highway in Mosul City
}

\author{
Yehia R. Hamdy Dr. Sami A. Mawjoud \\ Electrical Engineering Department \\ University of Mosul \\ Electrical Engineering Department \\ University of Mosul
}

\section{Abstract}

The aim of this paper is the assessment of the performance of U-TDOA Position Location (PL) method on a UMTS cellular system in Mosul city, Iraq. The study area is a $(3 \times 3 \mathbf{~ k m})$ which covers the University of Mosul campus. Simulation is used to study and evaluate the performance of U-TDOA PL method in urban area and on a highway inside the city. The effect of terrain, multipath, signal to noise ratio (SNR), Geometric Dilution Of Precision (GDOP), shadowing and the configuration of base stations on the positioning accuracy are investigated. The study is conducted on a real coordinates with the distribution of UMTS BSs as the same existing 2.5 Generation Enhanced Data rates for GSM Evolution (EDGE) Asiacell operator.

Keywords: U-TDOA, Position Location, UMTS.

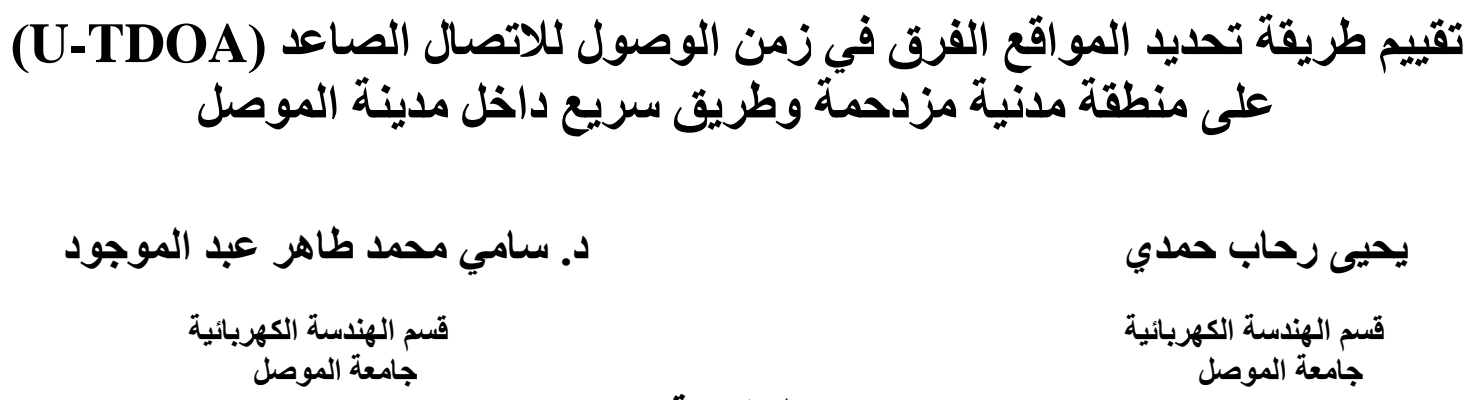

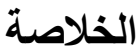

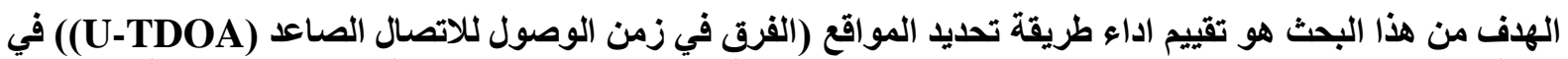

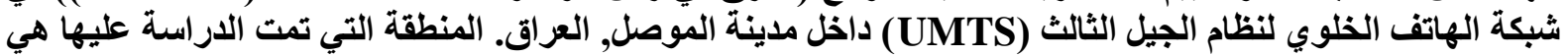

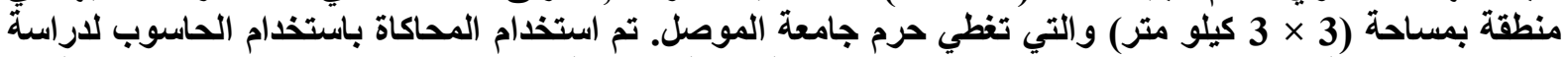

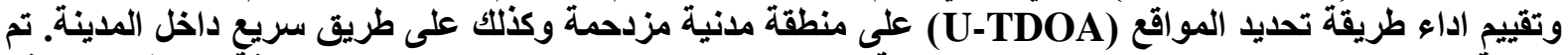

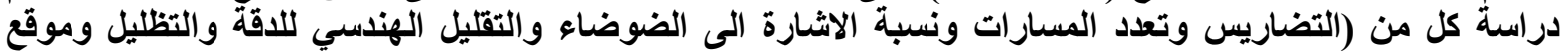

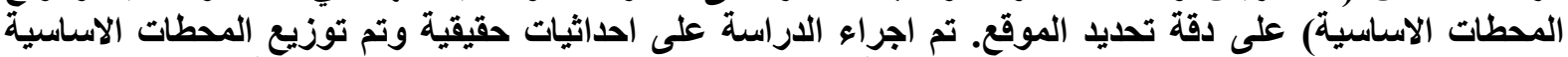
لنظام (UMTS) على نفس مواقع المحطات الاساسية لنظام الجيل الثاني المحسن (EDGE) لثركة اسياسيل.

Received: 17 - 10 - 2011

Accepted: 18 - 7 - 2012 


\section{Introduction}

The U-TDOA positioning method depends on Time Difference Of Arrival (TDOA) measurements. The measurements taken by at least three base stations to provide two dimensions (2D) position. The (Uplink) is used for referencing the Uplink channel and distinct it from Observed Time Difference Of Arrival (OTDOA) method, which works on the Downlink channel and depends also on (TDOA) measurements. Although both U-TDOA and OTDOA methods depend on (TDOA) measurements and both are applicable to UMTS, there are major differences between them, since U-TDOA is a network based positioning method, and OTDOA is a handset based positioning method [1].

OTDOA is the earliest version standardized by 3GPP for 3G (UMTS) by the end of the nineties through release99. This method works on Downlink channel and requires modification in both the infrastructure and handset, which require replacement of the legacy handset since the positioning calculation are done in the handset [2]. Another problem of this method is the hearability issue which is related to the closed loop power control that keeps the received power at the minimum required for the connection, and makes the handset unable to listen to other faraway base stations participating in the positioning process [3].

A new approach introduced which assumes modification to OTDOA method to overcome the hearability problem. The new approach assumes that the serving base station stops transmitting for a short period to allow the mobile to listen to other base stations far away from it which assist in the positioning process. The short period is called Ideal Period hence the name of Ideal Period Observed Time Difference Of Arrival (IP-OTDOA). The Ideal period length can vary, typically between 5 to $10 \mathrm{CPICH}$ symbols. Although this method overcomes the hearability problem, it has a major influence on the system capacity, which is the main concern in cellular systems [4].

U-TDOA is first proposed by TruePosition in USA. This method works on Uplink channel and the positioning calculation is done by the network without requiring modification to the handset. Also there is no hearability problem in this method since the mobile is usually monitored by two or more base stations for soft handover process. Only small modification to the infrastructure of the cellular system is required, thus Location Measurement Units (LMUs) should be planted at each base station which measures the time difference of arrived signal to extract the ranging equations. Then, these equations are applied to an algorithm for solving to give an estimated position fix [5].

The U-TDOA method is applicable for both GSM and UMTS, although it is specified for UMTS system, there is no earlier research study concerning the performance of U-TDOA PL method on UMTS system, it has been adopted by GSM operators in U.S. and serve about 100 million users [5].

\section{U-TDOA Positioning Estimation Techniques}

The U-TDOA system determines the mobile phone position based on trilateration, as shown in Figure 1. This system measures the time difference of the received signal from two base stations (e.g. BS1 and BS2) and convert this time difference to a constant difference distance between the two base stations (as foci) to define a hyperbolic curve. Other measurements between two base stations (e.g. BS1 and BS3) are considered to produce another hyperbola, the intersection of two hyperbolas determines the position fix [6]. 


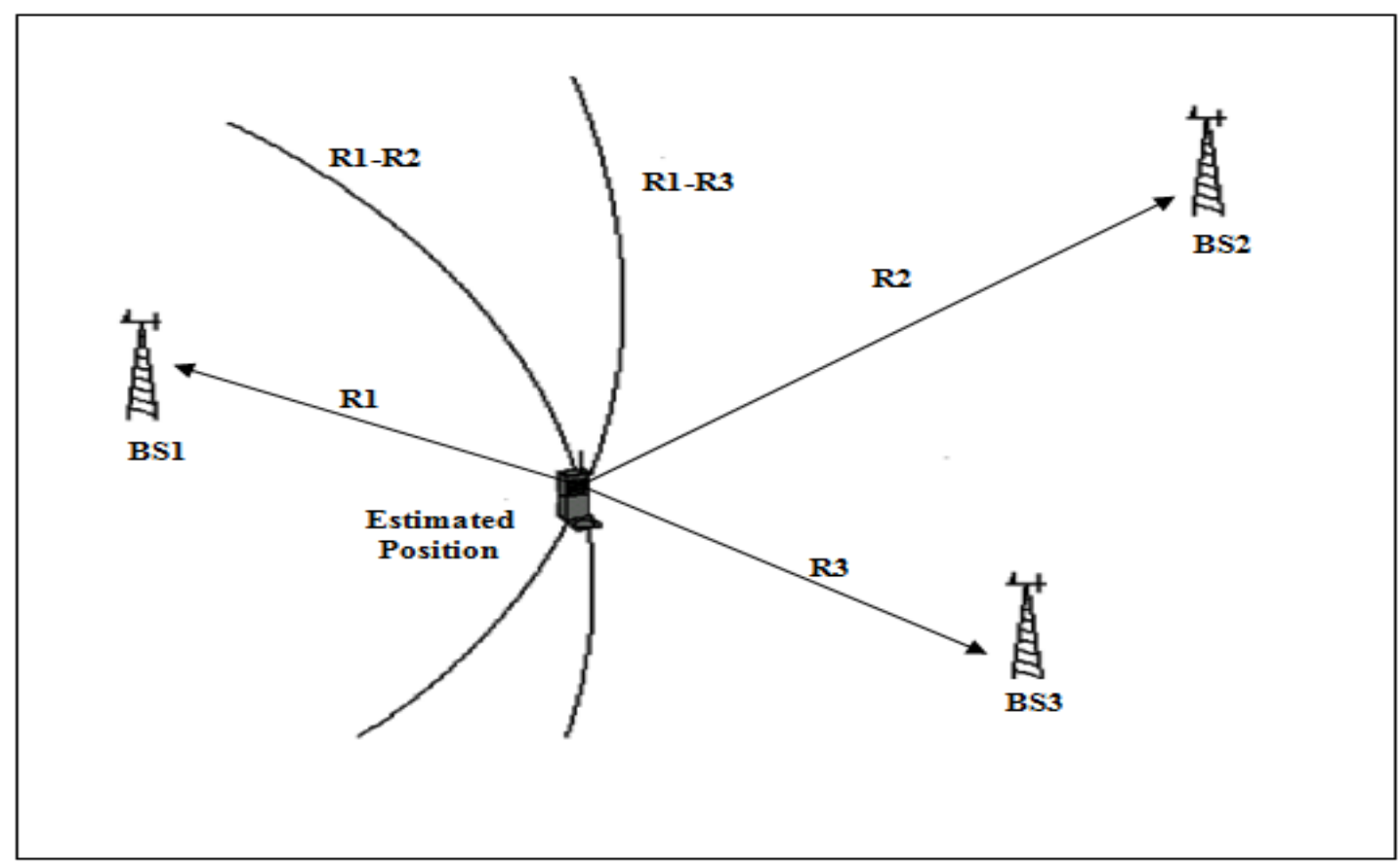

Figure (1) U-TDOA estimating method.

\section{Mathematical Model for U-TDOA}

A general model for two dimensional (2D) PL estimation of a mobile using M base stations is developed. Referring all U-TDOA's to the first base station, which is assumed to be the base station controlling the call and the first to receive the transmitted signal. Let the index $\mathrm{i}=2, \ldots, \mathrm{M}$, unless otherwise specified, $(\mathrm{x}, \mathrm{y})$ be the mobile location and $\left(\mathrm{X}_{\mathrm{i}}, \mathrm{Y}_{\mathrm{i}}\right)$ be the known location of the ith base station. The squared of the distance between the mobile and the ith base station is given as:

$$
\begin{aligned}
R_{i} & =\sqrt{\left(X_{i}-x\right)^{2}+\left(Y_{i}-y\right)^{2}} \\
& =\sqrt{X_{i}^{2}+Y_{i}^{2}-2 X_{i} x-2 Y_{i} y+x^{2}+y^{2}}
\end{aligned}
$$

The range difference between the two base stations with respect to the first base station where the signal arrives first, is[7]

$$
\begin{aligned}
R_{i, 1} & =c d_{i, 1}=R_{i}-R_{1} \\
& =\sqrt{\left(X_{i}-x\right)^{2}+\left(Y_{i}-y\right)^{2}}-\sqrt{\left(X_{1}-x\right)^{2}+\left(Y_{1}-y\right)^{2}}
\end{aligned}
$$

Where :

$\mathrm{c}$ is the signal propagation speed.

$R_{i, 1}$ is the range difference distance between the first base station and the ith base station.

$R_{1}$ is the distance between the first base station and the mobile.

$\mathrm{d}_{\mathrm{i}, 1}$ is the estimated U-TDOA between the first base station and the ith base station.

Equation (2) defines the set of nonlinear hyperbolic equations whose solution gives the $2 \mathrm{D}$ coordinates of the mobile [6]. 
There is no explicit solution to the nonlinear equations (2). Consequently, linearizing this set of equations is commonly performed. One way of linearizing these equations is through the use of Taylor-series expansion and retaining the first two terms [8][9]. A commonly used alternative method to the Taylor-series expansion method, presented in [10][11], is to first transform the set of nonlinear equations (2) into another set of equations. Rearranging the form of (2) into

$$
R_{i}^{2}=\left(R_{i, 1}+R_{1}\right)^{2}
$$

Equation (1) can now be rewritten as

$$
R_{i, 1}^{2}+2 R_{i, 1} R_{1}+R_{1}^{2}=X_{i}^{2}+Y_{i}^{2}-2 X_{i} x-2 Y_{i} y+x^{2}+y^{2}
$$

Subtracting (1) at $\mathrm{i}=1$ from (4) results in

$$
R_{i, 1}^{2}+2 R_{i, 1} R_{1}=X_{i}^{2}+Y_{i}^{2}-2 X_{i, 1} x-2 Y_{i, 1} y+x^{2}+y^{2}
$$

Where :

$\mathrm{X}_{\mathrm{i}, 1}$ and $\mathrm{Y}_{\mathrm{i}, 1}$ are equal to $\mathrm{X}_{\mathrm{i}}-\mathrm{X}_{1}$ and $\mathrm{Y}_{\mathrm{i}}-\mathrm{Y}_{1}$ respectively.

The set of equations in (5) are now linear with the mobile location (x,y) and the range of the first base station to the mobile R1 as the unknowns, and are more easily handled.

\section{Algorithms for Solving U-TDOA}

There are many algorithms that can be used for solving the nonlinear equations of $U$ TDOA to give a position fix, each with different complexity, accuracy and limitations. Choosing the best to fit depends on the requirements (e.g. accuracy, topography and fading).

\subsection{Taylor Series Algorithm}

An algorithm to obtain precise estimate at reasonable noise levels (suburban and rural areas where one of the received signal components is LOS) is the Taylor-series method [8][9]. The Taylor-series method linearizes the set of equations in (2) by Taylor-series expansion, and then uses an iterative method to solve the system of linear equations. The iterative method begins with an initial guess and improves the estimate at each iteration by determining the local linear least-square (LS) solution.

The Taylor-series method provides accurate results, however, it requires a close initial guess to guarantee convergence and can be computationally intensive.

\subsection{Fang's Algorithm}

For arbitrarily base stations locations and a number of equations equal the number of unknown mobile coordinates to be solved, Fang [12] provides an exact solution to equations (5).

This solution does not make use of redundant measurements made at additional base stations (more than three base stations) to improve position location accuracy. Also, this method experiences an ambiguity problem due to the inherent squaring operation. Furthermore, the unique assumption of base stations coordinates require modification of the actual coordinates of these base stations. This coordinates modification is difficult and 
undesirable in practical cellular environment because of the extra complexity added to the process [13].

\subsection{Chan's Algorithm}

A non-iterative solution to the U-TDOA position estimation problem, which is capable of achieving optimum performance for arbitrarily placed sensors, was proposed by Chan [14]. The solution is in closed-form and valid for both close and distant mobiles. For a three base stations system $(M=3)$, resulting in two U-TDOA's, $x$ and y can be solved in terms of R1 from (5). The solution is in the form of

$$
\left[\begin{array}{l}
x \\
y
\end{array}\right]=-\left[\begin{array}{ll}
X_{2,1} & Y_{2,1} \\
X_{3,1} & Y_{3,1}
\end{array}\right]^{-1} \times\left\{\left[\begin{array}{l}
R_{2,1} \\
R_{3,1}
\end{array}\right] R_{1}+\frac{1}{2}\left[\begin{array}{c}
R_{2,1}^{2}-K_{2}+K_{1} \\
R_{3,1}^{2}-K_{3}+K_{1}
\end{array}\right]\right\}
$$

Where :

$$
\begin{aligned}
& K_{1}=X_{1}^{2}+Y_{1}^{2} \\
& K_{2}=X_{2}^{2}+Y_{2}^{2} \\
& K_{3}=X_{3}^{2}+Y_{3}^{2}
\end{aligned}
$$

When equation (6) is inserted into equation (1), with $\mathrm{i}=1$, a quadratic equation in terms of R1 is produced. Substituting the positive root into (6) results in the final solution.

When Chan's algorithm is compared with Fang and Taylor-series algorithms discussed previously, it is seen that it is the best choice for solving the U-TDOA equations. Chan's algorithm gives exact solution and is better from accuracy point of view and less complex than the Taylor-series algorithm which is iterative and has the risk of convergence to local minima. When compared with Fang's algorithm, it is seen that it can take advantage of redundant measurements (made at more than three base stations), if available, whereas Fang's algorithm cannot. Also, Chan's algorithm works better than Fang's and Taylor-series under NLOS environment. Hence, Chan's algorithm is the best available option for solving UTDOA equations for positioning applications.

\section{Positioning Accuracy in U-TDOA Method}

There are many methods for measuring the accuracy of a 2D positioning methods that can be used to evaluate U-TDOA positioning method. Two methods are presented [7].

\subsection{Root Mean Square Error (RMS-Error)}

One of the simplest and efficient method for estimating the positioning accuracy is by measuring the RMS-Error, which is the distance in meter from the estimated position to the actual position. The mathematical model for RMS-Error starts by measuring the Mean Square Error (MSE) as[6][7]

$$
M S E=\varepsilon=\mathbf{E}\left[(x-\hat{x})^{2}+(y-\hat{y})^{2}\right]
$$


Where $(\mathrm{x}, \mathrm{y})$ is the coordinates of the mobile and $(\hat{\boldsymbol{x}}, \widehat{y})$ is the estimated position of the mobile. The Root Mean Square (RMS) Error is then computed

$$
R M S=\sqrt{\varepsilon}=\sqrt{\mathbf{E}\left[(x-\hat{x})^{2}+(y-\hat{y})^{2}\right]}
$$

\subsection{Cramer-Rao Lower Bound}

A commonly used measure of accuracy of a positioning method is to compare the Root Mean Squared RMS Error of the PL solution with the theoretical RMS Error based on the Cramer-Rao Lower Bound (CRLB) on the variance of unbiased estimator.

To gauge the accuracy of the PL estimator, the calculated RMS PL error is compared with the theoretical RMS error based on the Cramer-Rao Lower Bound (CRLB). The conventional CRLB sets a lower bound for the variance of any unbiased parameter estimator and is typically used for a stationary Gaussian signal in the presence of stationary Gaussian noise [15]. For non-Gaussian and non-stationary (cyclostationary) signals and noise, alternate methods have been used to evaluate the performance of the estimators. The CRLB on the PL covariance is given by Chan [14] as

$$
\mathbf{\Phi}=c^{2}\left(\mathbf{G}_{t}^{T} \mathbf{Q}^{-1} \mathbf{G}_{t}\right)^{-1}
$$

Where:

$$
\mathbf{G}_{t}=\left[\begin{array}{cc}
{\left[\left(X_{1}-x\right) / R_{1}\right]-\left[\left(X_{2}-x\right) / R_{2}\right]} & {\left[\left(Y_{1}-y\right) / R_{1}\right]-\left[\left(Y_{2}-y\right) / R_{2}\right]} \\
{\left[\left(X_{1}-x\right) / R_{1}\right]-\left[\left(X_{3}-x\right) / R_{3}\right]} & {\left[\left(Y_{1}-y\right) / R_{1}\right]-\left[\left(Y_{3}-y\right) / R_{3}\right]} \\
\vdots & \vdots \\
{\left[\left(X_{1}-x\right) / R_{1}\right]-\left[\left(X_{M}-x\right) / R_{M}\right]} & {\left[\left(Y_{1}-y\right) / R_{1}\right]-\left[\left(Y_{M}-y\right) / R_{M}\right]}
\end{array}\right]
$$

$\mathrm{Q}$ is the U-TDOA covariance matrix

The sum of the diagonal elements of $\Phi$ defines the theoretical lower bound on the RMS Error of the PL estimator. Matrix Q may not be known in practice; however, if the noise power spectral densities are similar at the receivers, it can be replaced by a theoretical U-TDOA covariance matrix with diagonal elements of $\sigma \mathrm{d} 2$ and $0.5 \sigma \mathrm{d} 2$ for all other elements, where $\sigma \mathrm{d} 2$ is the variance of the U-TDOA estimate [14].

\section{Factors Influencing the Accuracy and Performance of U-TDOA Method}

The accuracy of the U-TDOA positioning method is influenced by many factors each with different effect on the performance of the method, below a presentation of each [5][16].

- Signal to Noise Ratio (SNR)

- Bandwidth of the signal

- Multipath

- Geometric Dilution Of Precision (GDOP)

- Number of measurements

- Integration time 


\section{The Model Considered}

\subsection{Cellular System}

Model dealt with is an area of $(3 * 3 \mathrm{~km})$ which covers the University of Mosul campus. The plan of Asiacell GSM900 \& GSM1800 operator (BSs coordinates and towers heights) are applied on the map. The base stations of UMTS2100 used in simulation are distributed on the map with actual coordinates and heights of Asiacell towers. The coexciting GSM \& UMTS is usually used in practice [17]. This means that UMTS antennas share the GSM towers to reduce the cost and fast up the implementation of UMTS system.

The parameters of the cellular system used in simulation are presented in table 1 [17].

\section{2. $\quad$ Path Loss Fading}

The path loss model used in the simulation is Personal Communication Systems (PCS) Model [18][19]. This model is an Extension to Hata-Okumura Model [20] and it is an empirical formula. The European Co-operative for Scientific and Technical research (EUROCOST) formed the COST-231 working committee to develop an extended version of the Hata- Okumura model to make it suitable for Personal Communication Systems (PCS) which has radius of no more than $1 \mathrm{~km}$. The modified model extends Hata-Okumura model to $2 \mathrm{GHz}$ and valid for cell radius of less than $1 \mathrm{~km}$, and is given as :

$$
\begin{aligned}
\mathrm{L}(\text { urban })= & 46.3+33.9 \log \left(\mathrm{f}_{\mathrm{c}}\right)-13.82 \log \left(\mathrm{h}_{\mathrm{te}}\right)-\mathrm{a}\left(\mathrm{h}_{\mathrm{re}}\right) \\
& +\left(44.9-6.55 \log \left(\mathrm{h}_{\mathrm{te}}\right)\right) \log (\mathrm{d})+\mathrm{C}_{\mathrm{M}}
\end{aligned}
$$

Where :

$\mathrm{L}$ (urban) is the median path loss in $\mathrm{dB}$

$\mathrm{f}_{\mathrm{c}}$ is the carrier frequency in $\mathrm{MHz}$

$\mathrm{h}_{\mathrm{te}}$ is the effective base station antenna height in meter

$\mathrm{h}_{\mathrm{re}}$ is the effective mobile antenna height in meter

$\mathrm{d}$ is distance between mobile and base station in $\mathrm{km}$

$\mathrm{C}_{\mathrm{M}}$ equals $0 \mathrm{~dB}$ for medium sized city and suburban areas and $3 \mathrm{~dB}$ for metropolitan centers $\mathrm{a}\left(\mathrm{h}_{\mathrm{re}}\right)$ is the correction factor in $\mathrm{dB}$ for effective mobile antenna height which is a function of the size of the coverage area and given by[18]

$$
\mathrm{a}\left(\mathrm{h}_{\mathrm{re}}\right)=\left(1.1 \log \left(\mathrm{f}_{\mathrm{c}}\right)-0.7\right) \mathrm{h}_{\mathrm{re}}-\left(1.56 \log \left(\mathrm{f}_{\mathrm{c}}\right)-0.8\right)
$$

Table (1) Parameters of the cellular system.

\begin{tabular}{|c|c|}
\hline Parameter & value \\
\hline System & UMTS2100 \\
\hline Environment & $\begin{array}{c}\text { Urban area }(3 * 3 \mathrm{~km}) \text { PCS } \\
\text { microcellular system }\end{array}$ \\
\hline Number of cells (BSs) & 12 \\
\hline Cell radius & $500 \mathrm{~meter}$ \\
\hline Chip rate & $3.84 \mathrm{Mcps}$ \\
\hline Maximum power for uplink voice channel 12.2 kbps & $125 \mathrm{~dB}$ \\
\hline Frame length & $10 \mathrm{~ms}$ \\
\hline Required SNR for voice channel 12.2 kbps & $5 \mathrm{~dB}$ \\
\hline Processing gain for voice channel 12.2 kbps & $25 \mathrm{~dB}$ \\
\hline
\end{tabular}




\subsection{Multipath Fading}

Multipath fading refers to the dramatic changes in signal amplitude and phase that can be experienced as a result of small changes in distance (a small fraction of half-wavelength) in the spatial separation between a receiver and a transmitter. The received signal consists of large number of multiple paths. The general case of small scale multipath fading in the cellular systems obeys Rayleigh fading distribution where there is no line of sight signal component in the received signal [18][21].

Multipath fading has a special impact on positioning systems depending on time measurements, since $1 \mu \mathrm{s}$ error in time measurements leads to 300 meter error in position estimation.

\section{Simulation \\ 8.1. Urban Area}

For the urban area under consideration, forty position fix are studied with steps of $10 \%$ of the cell radius (50 meter). A path of $2 \mathrm{~km}$ inside the urban area, this path passes through different terrain and surroundings in order to simulate real cellular environment. The MS moves in different directions (curves) to represent urban area environment. At each position fix the MS requests for positioning from the network, the Base Station Controller (BSC) measure the received SNR using Uplink control channel which is received by all BSs in the area. The serving BS with the two best received SNR from BSs are chosen to participate in the positioning process. The reason behind choosing the best received SNR is to provide enough power for cross correlation to extract time difference measurements with minimum errors.

The three base stations selected for positioning process receive the same frame sent by the mobile station on the Uplink voice channel at a synchronized time. The received signal is delivered to the location server which is connected to the infrastructure of the cellular system to estimate the mobile position. The estimated mobile position is in linear coordinates UTM form, the location server transform the position into WGS-84 form to be applicable on maps. The estimated position is then sent via SMS, MMS or data link to the MS. The mobile downloads a map of the site from internet and pinpoint the position on the user screen.

\subsection{Highway}

For highway simulation, also a $2 \mathrm{~km}$ path with forty position fix in the area considered is studied. The idea is to simulate the movements of the MS in a straight direction with BSs laying on one side of the road. This configuration gives poor GDOP which results in poor accuracy of the positioning method. As in the urban area dealt with before, the positioning process starts with the BSC measuring the SNR on Uplink control channel for choosing the strongest BSs receiving the MS signal.

Once the three base stations with the best received SNR are chosen, the positioning process starts with the same steps discussed for urban area case.

\section{Results}

\subsection{Urban Area}

Figure 2 represents the results of simulation in urban area, it can be noticed that the worst position accuracy (RMS error $89.42 \mathrm{~m}$ ) is at position number 24 , the reason for this error is this position lays in a valley surrounded by three hills, which cause the signal of MS 
to suffer from sever multipath delay before reaching BSs. The best position accuracy (RMS error $27.77 \mathrm{~m}$ ) is at position number 5 which has low ambiguity region (low GDOP value) and suffer from low multipath delay.

The average RMS error for urban area is 51.69 meter which is acceptable value for positioning location methods specified by Federal Communication Commission (FCC) which is 100 meter error for emergency cases.

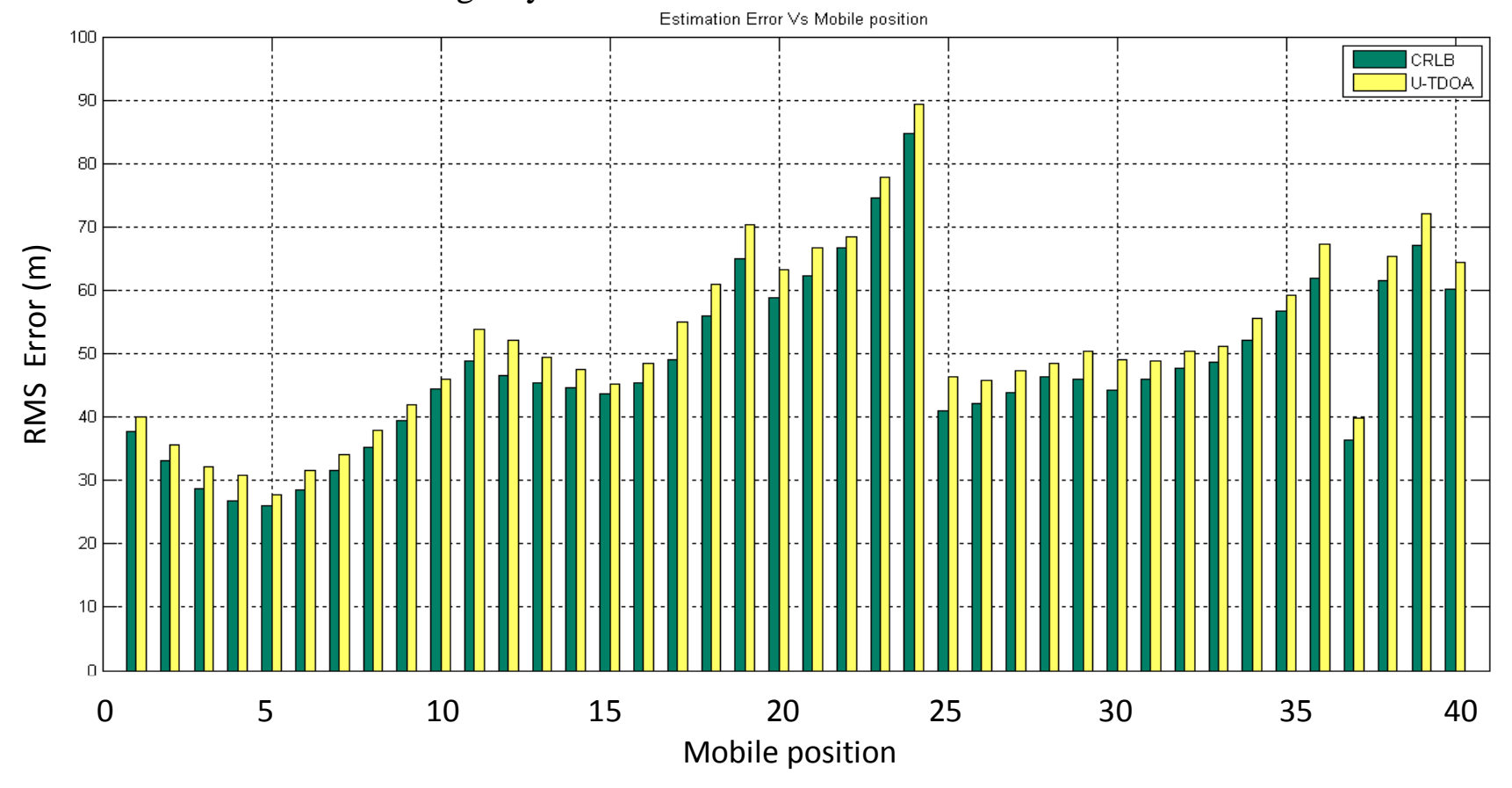

Figure (2) CRLB RMS Error and U-TDOA RMS Error vs. mobile position for urban area.

Figure 3 shows the positions of the MS for $2 \mathrm{~km}$ path in urban area (green pinpoints are true position of MS, red pinpoints are estimated U-TDOA positions of MS and yellow pinpoints are the BSs).
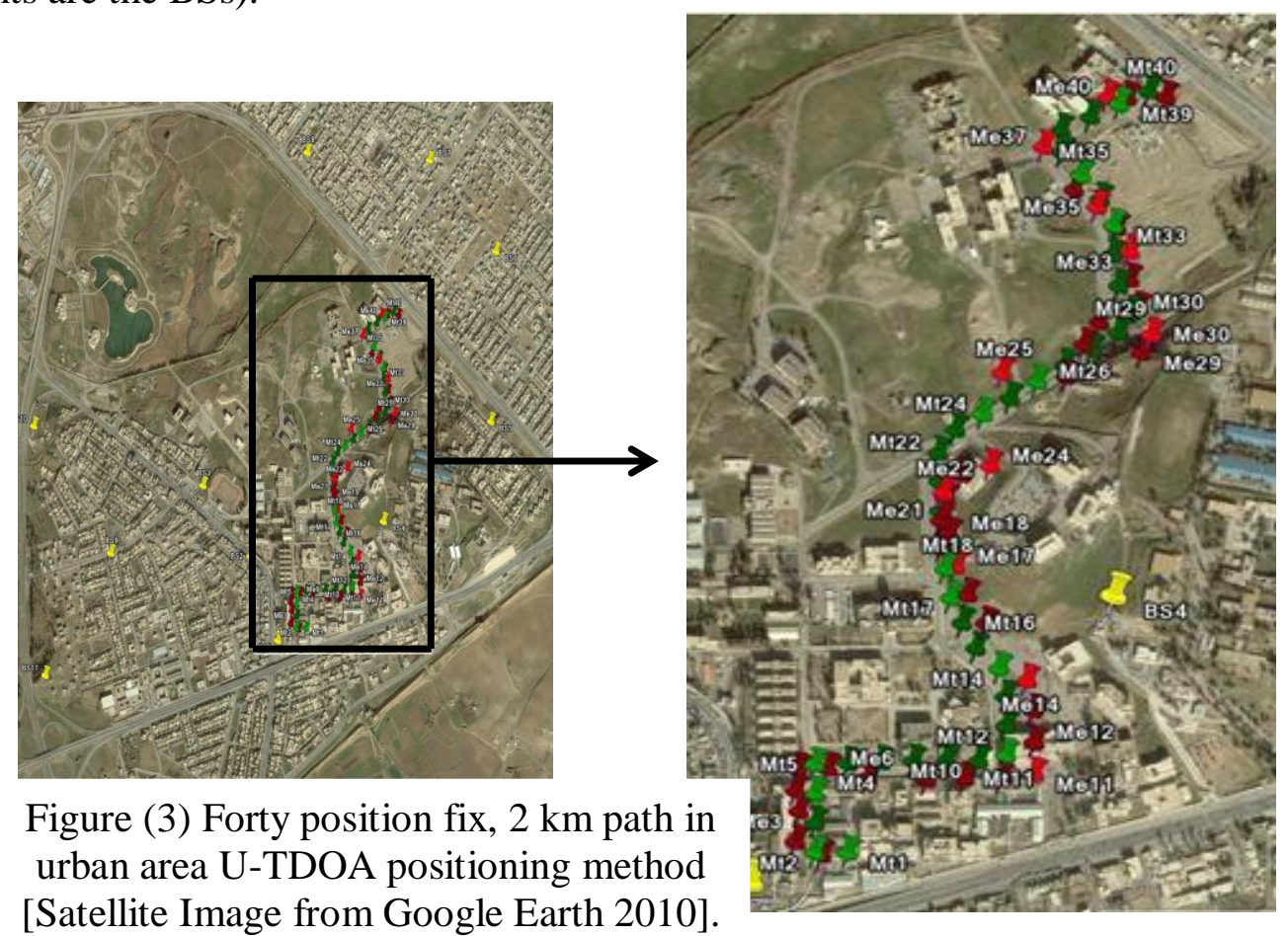

Figure (3) Forty position fix, $2 \mathrm{~km}$ path in urban area U-TDOA positioning method [Satellite Image from Google Earth 2010]. 


\subsection{Highway}

Figure 4 represents the results of simulation in highway, it can be observed that the worst position accuracy (RMS error $898.33 \mathrm{~m}$ ) is at position number 21, the reason for this high error is that the GDOP of this position is very high, which makes the ambiguity (uncertainty) of this position is very high, implying that even any low multipath delay can cause highly error in position. In this case, the error represents the limitation of using PL methods with bad GDOP on highways.

The best position accuracy (RMS error $26.49 \mathrm{~m}$ ) is at position number 8 which has good GDOP configuration (BSs surround MS from both sides of the road) making low ambiguity region for estimation of MS.

Although some extreme cases that happened on highway due to bad GDOP, the overall performance of U-TDOA method is acceptable with average RMS error $86.3 \mathrm{~m}$, which is under the required PL error specified by FCC.

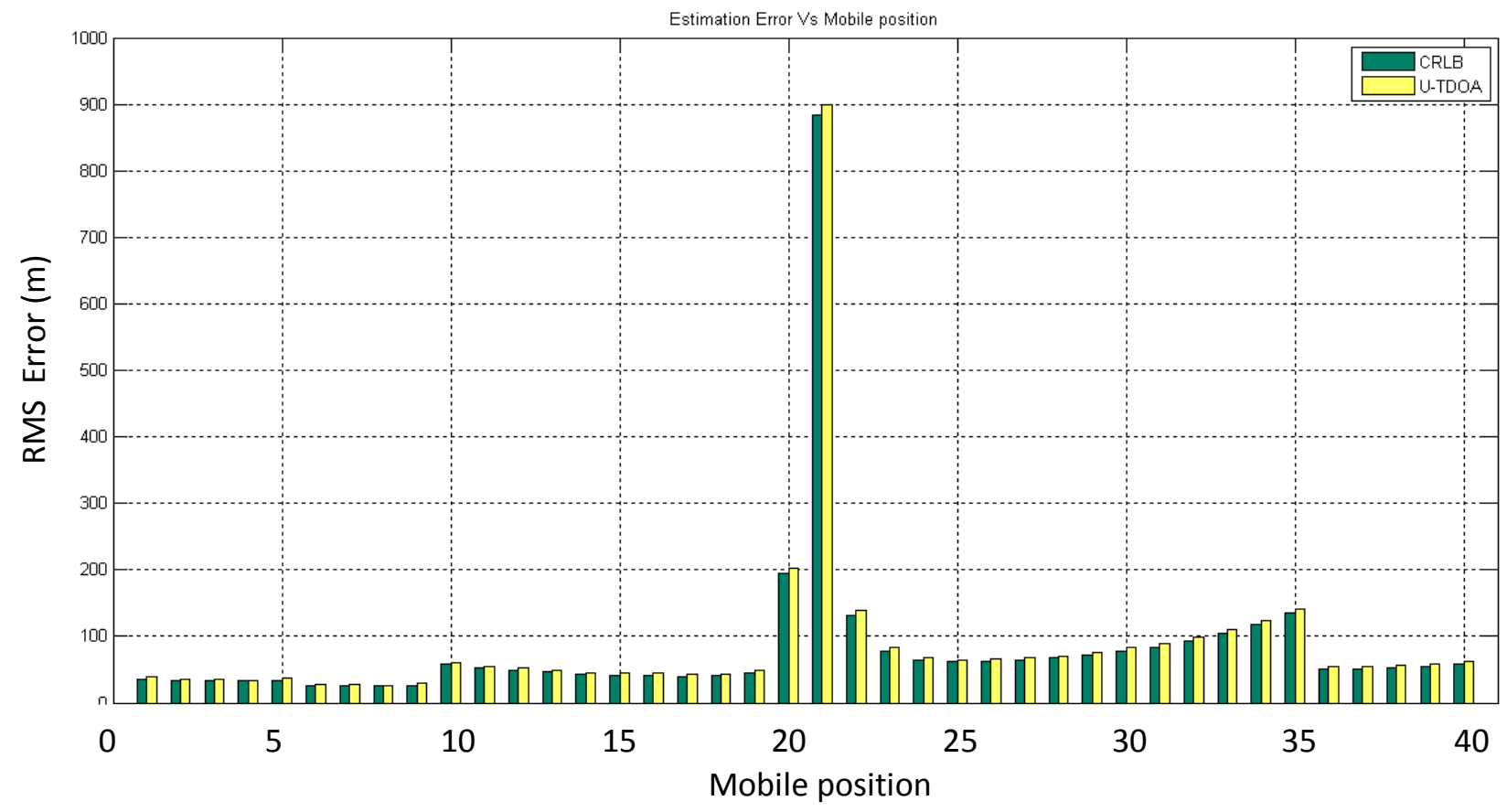

Figure (4)CRLB RMS Error and U-TDOA RMS Error vs. mobile position for highway

Figure 5 shows the positions of MS for $2 \mathrm{~km}$ path in highway (green pinpoints are true position of MS, red pinpoints are estimated U-TDOA positions of MS and yellow pinpoints are the BSs). 


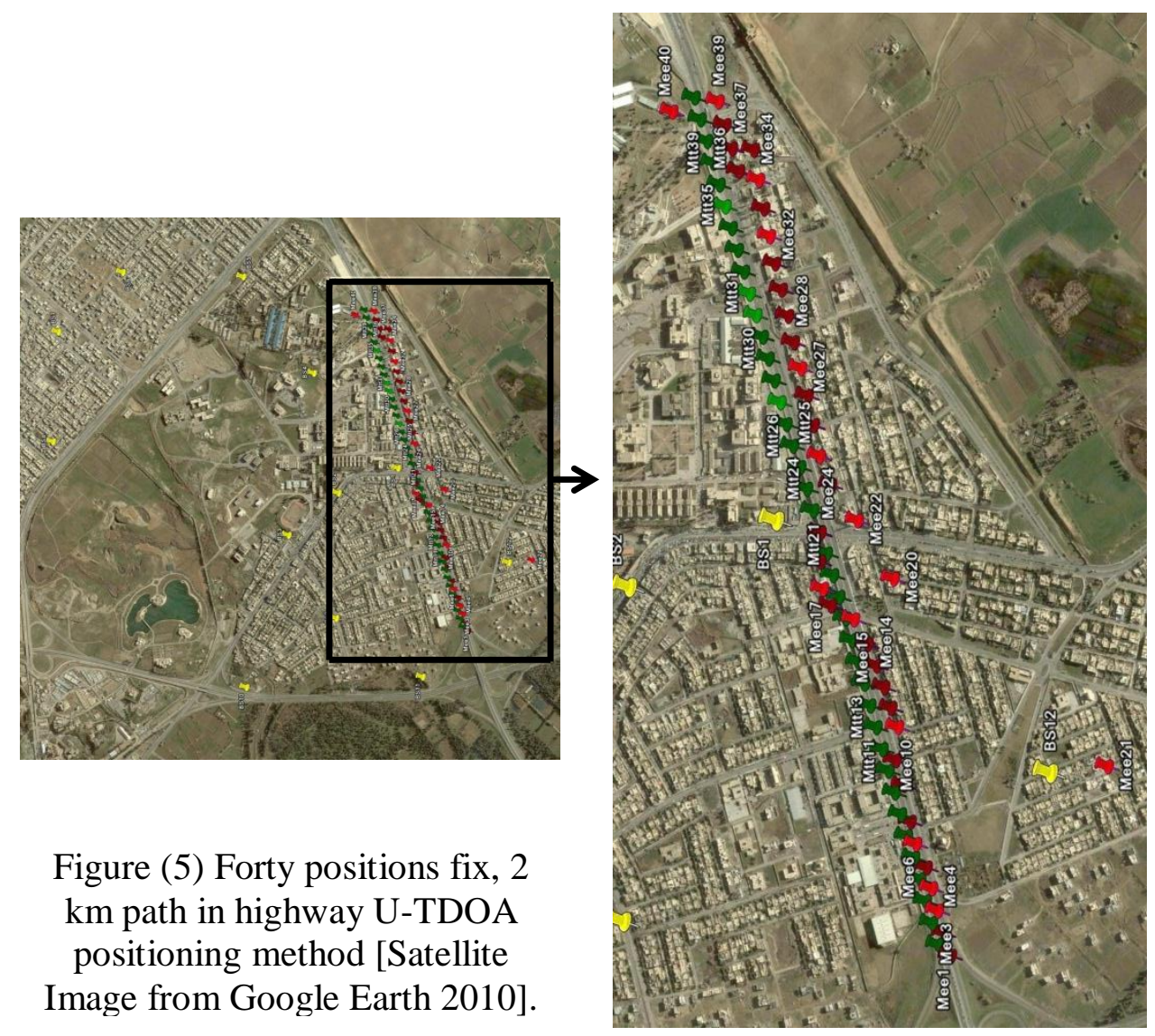

\section{Conclusions}

The geometry of BSs and MS has a significant influence on PL method performance. This geometry is known as the Geometric Dilution of Precision (GDOP). The GDOP differs in urban area (good GDOP) from highway (poor GDOP), the reason for the difference is that in urban area the BSs surrounds MS which gives good GDOP and hence better accuracy, while on highway the BSs lay on one side of the road which results in poor GDOP and hence lower accuracy.

The accuracy of U-TDOA PL method in urban area is highly affected by multipath propagation due to the obstacles present such as buildings, hills, canyons, etc. that shadows the LOS propagation of the cellular signals, this effect is present in simulation.

On highway, the GDOP is poor, the accuracy of U-TDOA PL method can be affected severely due to multipath propagation delay. The reason for that is in poor GDOP environment the ambiguity region is very large, hence even a small multipath present can lead to a very high error in position.

The accuracy of U-TDOA PL method in urban area is very good with average RMS error of 51.69 meter. The accuracy of U-TDOA PL method on the highway is less compared to the accuracy of U-TDOA PL method in urban area, but still works well as average RMS error of 86.3 meter which is less than the RMS error of 100 meter recommended by FCC for emergency cases. Although a few odd cases where the method failed to fulfill the requirements of FCC accuracy due to poor GDOP. 


\section{References}

[1] A. Roxin, J. Gaber, M. Wack and A. Nait-Sidi-Moh, " Survey of Wireless Geolocation Techniques", Globecom workshops, Washington, DC, Issue Date: 26-30 Nov. 2007, IEEE.

[2] Y. Zhao, " Standardization of Mobile Phone Positioning for 3G Systems", IEEE Communications Magazine, vol. 40, pp. 108-116, July 2002.

[3] D. Porcino, " Location of Third Generation Mobile Devices: A Comparison between Terrestrial and Satellite Positioning Systems", IEEE Vehicular Technology Conference, Rhodes , Greece, Meeting Date: 6-9 May 2001, vol. 4, pp. 2970-2974.

[4] S. Ahonen, J. Lahteenmaki, H. Laitinen and S. Horsmanheimo, " Usage of Mobile Location Techniques for UMTS Network Planning in Urban Environment", in Proceedings of the IST Summit 2002, pp. 823-827, 2002.

[5] J. F. Bull, " Wireless Geolocation", IEEE Vehicular Technology Magazine, vol. 4, pp. 45-53, December 2009, IEEE.

[6] Y. R. Hamdy, S. A. Mawjoud, " Performance Assessment of U-TDOA and A-GPS Positioning Methods", International Conference on Future Communication Networks, Baghdad, Iraq, pp. 99-104, Date: 10-12 April 2012, IEEE.

[7] Y. R. Hamdy, " Assessment of Mobile Position Location Methods in Cellular Systems", M.Sc. Thesis, University of Mosul, Mosul, Iraq, April 2011.

[8] W. H. Foy, " Position-Location Solutions by Taylor-Series Estimation", IEEE Transactions on Aerospace and Electronic Systems, vol. AES-12, pp. 187-194, March 1976.

[9] D. J. Torrieri, " Statistical Theory of Passive Location Systems", IEEE Transactions on Aerospace and Electronic Systems, vol. AES-20, pp. 183-198, March 1984.

[10] B. Friedlander, " A Passive Localization Algorithm and Its Accuracy Analysis", IEEE Journal of Oceanic Engineering, vol. 12, pp. 234-245, January 1987.

[11] H. C. Schau and A. Z. Robinson, " Passive Source Localization Employing Intersecting Spherical Surfaces from Time-of-Arrival Differences", IEEE Transactions on Acoustics, Speech and Signal Processing, vol. 35, pp. 1223-1225, August 1987.

[12] B. T. Fang, "Simple Solutions for Hyperbolic and Related Position Fixes", IEEE Transactions on Aerospace and Electronic Systems, vol. 26, pp. 748-753, September 1990.

[13] Y. Suman, A. Nistads, K. S. Marg and N. Rajpal, " Analysis and Simulation of Mobile Location Tracking Techniques", IETE Journal of Research, Vol 54, No. 1, JanuaryFebruary 2008, pp 51-60.

[14] Y. T. Chan, K. C. Ho, " A Simple and Efficient Estimator for Hyperbolic Location", IEEE Transactions on Signal Processing, vol. 42, pp. 1905-1915, Aug. 1994.

[15] C. Chen and W. A. Gardner, " Signal Selective Time-Difference-of-Arrival Estimation for Passive Location of Man-Made Signal Sources in Highly Corruptive Environments, Part II: Algorithms and Performance", IEEE Transactions on Signal Processing, vol. 40, pp. 1185-1197, May 1992.

[16] TruePosition, " U-TDOA Enabling New Location-based Safety and Security Solutions", White Paper, USA, October 2008.

[17] H. Holma and A. Toskala, " WCDMA for UMTS HSPA Evolution and LTE", Fifth Edition, John Wiley and Sons Ltd. Publication, England, 2010.

[18] T. S. Rappaport, "Wireless Communications Principles and Practice", Second Edition, Prentice-Hall Inc. Publication, USA, 2002.

[19] M. P. Green and S. S. Wang, " Signal Propagation Model Used to Predict Location Accuracy of GSM Mobile Phones for Emergency Applications", IEEE Radio and Wireless Conference, Date: 11-14 Aug. 2002, pp. 119-122.

[20] M. Hata, " Empirical Formula for Propagation Loss in Land Mobile Radio Services", IEEE Transactions on Vehicular Technology, Date: August 1980, vol. 29, pp. 317-325.

[21] J. Laiho, A. Wacker and T. Novosad, " Radio Network Planning and Optimisation for UMTS", Second Edition, John Wiley and Sons Ltd. Publication, England, 2006. 